\title{
Backstepping Output Feedback Control for the Stochastic Nonlinear System Based on Variable Function Constraints with the Subsea Intelligent Electroexecution Robot System
}

\author{
Long-Chuan Guo $\mathbb{D}^{1},{ }^{1}$ Jing $\mathrm{Ni}^{1}{ }^{1}$ Jing-Biao Liu, ${ }^{1}$ Xiang-Kun Fang, ${ }^{2}$ Qing-Hua Meng, \\ and Yu-Dong Peng ${ }^{1}$ \\ ${ }^{1}$ School of Mechanical Engineering, Hangzhou Dianzi University, Hangzhou 310018, China \\ ${ }^{2}$ Beijing Shougang International Engineering Technology Co. Ltd, Beijing 100043, China \\ Correspondence should be addressed to Long-Chuan Guo; glc1988@126.com
}

Received 30 September 2020; Revised 6 April 2021; Accepted 19 May 2021; Published 28 May 2021

Academic Editor: Sergey Dashkovskiy

Copyright (c) 2021 Long-Chuan Guo et al. This is an open access article distributed under the Creative Commons Attribution License, which permits unrestricted use, distribution, and reproduction in any medium, provided the original work is properly cited.

\begin{abstract}
The output feedback controller is designed for a class of stochastic nonlinear systems that satisfy uncertain function growth conditions for the first time. The multivariate function growth condition has greatly relaxed the restrictions on the drift and diffusion terms in the original stochastic nonlinear system. Here, we cleverly handle the problem of uncertain functions in the scaling process through the function maxima theory so that the Ito differential system can achieve output stabilization through Lyapunov function design and the solution of stochastic nonlinear system objects satisfies the existence of uniqueness, ensuring that the system is globally asymptotically stable in the sense of probability. Furthermore, it is concluded that the system is inversely optimally stable in the sense of probability. Finally, we apply the theoretical results to the practical subsea intelligent electroexecution robot control system and obtain good results.
\end{abstract}

\section{Introduction}

In the field of control theory, research on the output feedback control of stochastic nonlinear systems has produced some valuable results. As far as the output feedback control is concerned, it has obvious advantages over the state feedback control in practical systems because it does not need to obtain all the system states' information [1-10], and as far as the stochastic nonlinear system itself is concerned, stochastic process and nonlinearity constitute the two most basic characteristics of the practical system. As a practical system, external interference is inevitable, and many interferences appear as the stochastic process. This has obvious manifestations in the fields of engineering technology and socioeconomic management and even extends to the field of large systems. Therefore, the study of stochastic nonlinear systems has strong practical significance.

Krstić and Kanellakopoulos [11] studied the design of output feedback controllers for a class of stochastic nonlinear systems (1). The nonlinear terms in this system are all functions that can measure the output for the first time.

$$
\begin{aligned}
\mathrm{d} x_{i} & =x_{i+1} \mathrm{~d} t+\phi_{i}(y) \mathrm{d} \omega, \quad(i=1, \ldots, n-1), \\
\mathrm{d} x_{n} & =u \mathrm{~d} t+\phi_{n}(y) \mathrm{d} \omega, \\
y & =x_{1},
\end{aligned}
$$

where $\phi_{i}(y)$ is a smooth nonlinear function on the output, initial value $\phi_{i}(0)=0$, and $\omega$ is the standard Wiener process. In this paper, authors Deng and Krstic propose a controller based on full-order observers with the help of backstepping technology. The output feedback stabilization results of the 
system are given. Based on [6], a series of research results are subsequently produced in the research [11-22]. Lei and Lin [13] studied the output feedback stabilization of the system by changing the energy supply function method. Chang et al. [14] introduced a stochastic small-gain theorem to obtain a design scheme for output feedback controllers based on reduced-order observers. The above papers have one thing in common: their nonlinear terms all depend on measurable output terms.

For the controller design of high-order nonlinear systems, some research results have been obtained in recent years [23-33]. In early papers [20, 21], the authors used high-order nonlinear systems as strict feedback systems, based on which the controller design work and homogeneous dominant method have been proposed to effectively control the nonlinear terms. In subsequent papers [23,24], the authors continued to study output tracking and disturbance suppression for higherorder systems. Regarding the form of the controller, Mazenc et al. [25] used an increased power integration method to design a state feedback controller for high-order nonlinear systems which find the upper bound of the nonlinear term can make the closed-loop system globally asymptotically stable. The authors in [26-31] gradually weakened growth conditions of the nonlinear term and accordingly constructed smooth state feedback controllers. Deng and Krstic [26] studied high-order nonlinear systems with parameter uncertainty. With adaptive control methods, a dynamic gain adaptive law has been introduced, and an adaptive state feedback controller has been designed. Aiming at the problem of designing output feedback controllers for high-order nonlinear systems, especially the unmeasurability of states, first, a state observer needs to be built. Compared with the design of state feedback controllers, the problem of the output feedback control is very difficult.

In [28], for the output feedback design process of nonlinear systems, whether stability design or tracking control design, it is necessary to restrict the growth conditions of unmeasurable states in the nonlinear term; otherwise, there is no discussion of control issues. Nonlinear growth conditions are a hypothetical limitation on the nonlinear term, which are within a certain constraint. For example, there is a constant $C$ that makes the nonlinear term $f(x)$ satisfy $|f(x)| \leq C\left(\left|x_{1}\right|+\cdots+\left|x_{n}\right|\right)$ which is a typical linear growth condition. When the states' variable is finitely changed, the states have a finite increase as a nonlinear function of the independent variable, and the rate of growth satisfies certain constraints. As the most basic linear growth condition, this growth condition has been used by quite a lot of research results as the basic assumption for controller design [33-35]. For later researchers, there is a very challenging task for nonlinear term constraints, that further weakens the constraints on nonlinear terms. The latest research hotspots focus on how to design controllers in cases where nonlinear terms satisfy the power growth condition. In the early stage, for a nonlinear system that satisfies certain growth conditions, the state variables of the system itself are mostly measurable or partially measurable. However, in the practical system, the "black box" situation of the system is ubiquitous. In the case that the input and output are measurable and the state variables are unmeasurable, the design of the system has very practical value. In [28-32], the state feedback and output feedback control problems of nonlinear systems with unmeasurable states have been studied, and the constraints of nonlinear terms have been gradually relaxed.

Jiang and Praly [32] used homogeneous dominance theory and relaxed the growth conditions to design an output feedback controller. After introducing stochastic factors into higherorder nonlinear systems, some research results have been produced. Some of the results are mainly focused on the study of a special kind of stochastic nonlinear systems. The system is characterized by its nonlinear terms not only relying on measurable outputs but also on all unmeasurable states. Li et al. [34] studied a class of high-order stochastic nonlinear systems, which can neither feedback linearize nor affine to control inputs. The authors used the coordinate transformation method in nonlinear theory to construct a state observer for a stochastic nonlinear system, selected an appropriate Lyapunov function, and designed an output feedback controller which satisfies the system's stabilization conditions. $\mathrm{Li}$ et al. [35] further studied the stabilization of the output feedback for higher-order stochastic nonlinear systems with the relaxation of nonlinear term growth conditions. By using the design idea of the homogeneous dominant method, the final output feedback controller is obtained so that closed-loop stochastic nonlinear systems are globally asymptotically stable in the sense of probability. In recent years, the field of stochastic nonlinear control theory has focused on the study of a special kind of stochastic nonlinear systems, that is, the nonlinear terms of stochastic nonlinear systems depend on measurable outputs [35-48]. Liu and Xie [36] used the theory of homogeneity and the method of increasing power integration to construct an output feedback controller to ensure the global asymptotic stability of the system in the sense of probability. In [37], a state observer is constructed by introducing coordinate transformation, and the Lyapunov function is selected to study the design of output feedback controllers for stochastic nonlinear systems in a more general condition. Based on [36-41], Xie and Liu [38] removed the assumption that the observer gain is greater than one and avoided the conservative situation of the designed output feedback controller. Guo and Fang [39] introduced unknown control coefficients, and the values of the upper and lower bounds of the control coefficients are generalized by constants to allow arbitrary values to be arbitrarily large or arbitrarily small.

However, in the previous research results, the nonlinear terms (drift term and diffusion term) of the system must satisfy the growth condition which is linear and highly restrictive. This harsh condition makes the application of research results narrow. Therefore, this paper creatively proposes a more relaxed constraint condition, that is, a power growth condition $p(x)$ that has always been presented in the form of a function as follows:

$$
\left|\varphi_{i}(x)\right| \leq d\left(\left|x_{1}\right|^{p_{i}(t, x)}+\left|x_{2}\right|^{p_{i}(t, x)}+\cdots+\left|x_{i}\right|^{p_{i}(t, x)}\right) .
$$

This paper focuses on the output feedback problem of a class of stochastic nonlinear systems that satisfy the growth conditions of the form of an uncertain function. The 
nonlinear term depends on the measurable output and all unmeasurable states. For the output feedback problem of such systems, there is no latest research result. We strive to design the output feedback controller to ensure that the equilibrium point of the system's origin is globally asymptotically stable in the probabilistic sense. At the same time, we further derive that the system is inversely optimally stable in the probabilistic sense. The main contributions of this paper are as follows:

(1) The set power growth condition relaxes the restrictions on the drift and diffusion terms of the stochastic nonlinear system, and the scope of the application of the output feedback controller is also greatly expanded.

(2) In the previous research results, most of the systems studied are for the system determined by the constant growth condition. Even for uncertain systems, the control function has the control direction and control coefficient [33-35]. Li et al. [35] focused on the output feedback practical tracking problem for a class of stochastic nonlinear systems in which the coefficient in front of the control action growth condition $p(x)$ is 1 . Such system design greatly limits the scope of design and application of the system controller, especially in the actual system, because in many practical systems, such as aerospace systems, terrestrial satellite navigation systems, industrial processes, and other complex systems, the control role mostly contains uncertain factors. The uncertainty here does not only refer to the uncertainty of the system itself but also to the uncertainty of the system control. Therefore, it is urgent to study the uncertainty function growth condition. Based on this consideration, we consider the output feedback problem for a class of stochastic nonlinear systems with the uncertainty function growth condition. By introducing a new high-gain adjustment law, the design method of the global output control for such uncertain nonlinear systems is given.

(3) In [41], the authors proposed a design idea for a highgain observer. We use this design method to construct a state observer for a system object that satisfies the more general condition of the power growth condition. And by setting a series of Lyapunov functions and using the backstepping method, finally authors can get an output feedback controller with high-gain parameters so that the solution of the random nonlinear system object satisfies the existence of uniqueness while ensuring that the system is globally asymptotically stable in probability.

This paper is organized as follows. Section 2 provides the problem description. In Section 3, an output feedback controller is designed. In Section 4, the system is analyzed, and inverse optimal stabilization in probability is achieved. Section 5 applies theoretical results to the practical subsea electrohydraulic valve control system. Section 6 summarizes this paper.

\section{Problem Description}

This paper mainly studies the following types of stochastic nonlinear systems:

$$
\left\{\begin{array}{l}
\mathrm{d} x_{i}=x_{i+1} \mathrm{~d} t+\varphi_{i}(x) \mathrm{d} \omega, \quad(i=1, \ldots, n-1) \\
\mathrm{d} x_{n}=u \mathrm{~d} t+\varphi_{n}(x) \mathrm{d} \omega \\
y=x_{1}
\end{array}\right.
$$

In this stochastic nonlinear system, $x=\left(x_{1}, \ldots, x_{n}\right)^{T} \in R^{n}$ is the state of the system, $u \in R$ is the input of the system, and $y \in R$ is the output of the system. Observable state $x_{2}, \ldots, x_{n}$ is not measurable, and output $y$ is measurable. $\omega$ is an $m$-dimensional standard Brownian motion defined on the probability space $(\Omega, \Gamma, P)$. The nonlinear term $\varphi_{i}(i=1, \ldots, n): R^{n} \longrightarrow R^{m}$ is a continuous smooth function and satisfies $\varphi_{i}(0)=0$.

For the above system (3), the following assumptions are given.

Assumption 1. For the nonlinear term of system (3), there exists a constant such that the following inequality holds:

$$
\left|\varphi_{i}(x)\right| \leq d\left(\left|x_{1}\right|^{p_{i}(t, x)}+\cdots+\left|x_{i}\right|^{p_{i}(t, x)}\right),
$$

where $p_{i}(x, t)$ is a positive function.

Remark 1. In the previous research results, the constraints of the nonlinear term are very narrow, and the scope of the application is very small. Earlier constraints focused on linear growth conditions or piecewise linear growth conditions. In the latest part of the research, power growth conditions are applied to controller design, but there are still obvious shortcomings, that is, power growth conditions are basically definite constants, and this kind of constant is simply to increase the order which the system itself has not been greatly expanded. Then, in this paper, the determined power order value is creatively functionalized, that is, a variable function $p_{i}(x, t)$ is generated in a nonlinear constraint condition. Based on this, the entire stochastic nonlinear system has greatly increased adaptability.

In the following, we will introduce the design idea of the output feedback controller in detail. The final output feedback controller ensures that stochastic nonlinear system (3) is globally asymptotically stable in the sense of probability.

\section{Control Design}

The controller design of stochastic nonlinear system (3) is divided into two parts. The first part is to design a state observer for the system; the second part uses the backstepping method to design an output feedback controller for system (3) to meet the corresponding control performance indicators.

3.1. High-Order Gain Observer Design. Since the system is not measurable except for $x_{1}$ which is a measurable state, a set of high-order gain observers is first constructed: 


$$
\left\{\begin{array}{l}
\dot{\hat{x}}_{i}=\widehat{x}_{i+1}+K^{i} h_{i}\left(x_{1}-\widehat{x}_{1}\right), \quad(i=1, \ldots, n-1), \\
\dot{\hat{x}}_{n}=u+K^{n} h_{n}\left(x_{1}-\widehat{x}_{1}\right) .
\end{array}\right.
$$

In this set of observers, the real number $K>0$ is the highgain parameter to be designed, $\widehat{x}_{i}(i=1, \ldots, n)$ is dependent on the measurable output $y$, and $h_{i}>0$ is a known constant that stabilizes the matrix

$$
A=\left(\begin{array}{cc}
-h_{1} & \\
\cdots & I_{n-1} \\
-h_{n} & 0 \cdots 0
\end{array}\right) .
$$

Then, there exists a positive definite matrix $P$ that satisfies $A^{T} P+P A=-I$. Based on the above analysis, combined with the state observer, we define the error equation of system (3) as

$$
\begin{aligned}
\tilde{x} & =\left(\tilde{x}_{1}, \ldots, \tilde{x}_{n}\right), \\
\tilde{x}_{i} & =\frac{\left(x_{i}-\widehat{x}_{i}\right)}{K^{i-1}} \quad(i=1, \ldots, n) .
\end{aligned}
$$

According to (3)-(7), the system error model can be obtained as

$$
\mathrm{d} \tilde{x}=K A \tilde{x} \mathrm{~d} t+\Phi(x) \mathrm{d} \omega
$$

where

$$
\Phi(x)=\left(\varphi_{1}(x), \frac{\varphi_{2}(x)}{K}, \ldots, \frac{\varphi_{n}(x)}{K^{n-1}}\right)^{T} .
$$

Since the growth conditions of the system are controlled by the function group $p_{i}(x, t)$ with uncertain parameters, the key to the design of the output feedback control system in this paper is to handle the function growth conditions, normalize them, and limit the range. First, based on the output feedback control theory and its mathematical application, a Lyapunov function is selected:

$$
V_{0}(\widetilde{x})=(n+1) \tilde{x}^{T} P \widetilde{x}
$$

Use the following inequality:

$$
\begin{aligned}
(a+b)^{2} & \leq 2\left(a^{2}+b^{2}\right), \\
\sum_{i=1}^{n}\left|a_{i}\right|^{2} & \leq\left(\sum_{i=1}^{n}\left|a_{i}\right|\right)^{2}, \\
\left(\sum_{i=1}^{n} a_{i}\right)^{2} & \leq n \sum_{i=1}^{n} a_{i}^{2} .
\end{aligned}
$$

We can get the system's Ito differential equation based on this Lyapunov function as

$$
\begin{aligned}
L V_{0} \leq & -(n+1) K|\widetilde{x}|^{2}+(n+1)\|P\| d^{2}\left(\sum_{i=1}^{n} \frac{1}{K^{i-1}}\right)^{2}\left(\left|x_{1}\right|^{p_{1}(x)}+\cdots+\frac{\left|x_{n}\right|^{p_{n}(x)}}{K^{n-1}}\right) \\
\leq & \sqrt{\left|p_{1}(x)\right|+\cdots+\left|p_{n}(x)\right|} d^{*}\left(\sum_{i=1}^{n}\left(\frac{\widehat{x}_{i}^{\bar{\theta}}}{K^{i-1}}\right)+\sum_{i=1}^{n}\left(K \tilde{x}_{i}^{\bar{\theta}}\left|\bar{p}_{i}(x)\right|\right)\right)^{2}-(n+1) K|\widetilde{x}|^{2} \\
\leq & -\sqrt{\left|p_{1}(x)\right|+\cdots+\left|p_{n}(x)\right|}\left((n+1) n K-n d^{*} \sum_{i=1}^{n}\left(K \widetilde{x}_{i}^{\bar{\theta}}\left\|\bar{p}_{i}(x)\right\|\right)^{2}\right)\|\widetilde{x}\|_{\infty}^{2 \bar{\theta}} \\
& +n d\left(\hat{x}_{1}^{2 \bar{\theta}}+\frac{\hat{x}_{2}^{2 \bar{\theta}}}{K^{2}}+\cdots+\frac{\hat{x}_{n}^{2 \bar{\theta}}}{K^{2 n-2}}\right)
\end{aligned}
$$

where

$$
\begin{aligned}
\theta_{i} & =\left(\sqrt{p_{i \max }^{2}(x)+p_{i \min }^{2}(x)}\right), \\
\bar{\theta} & =\left|\theta_{1}\right|+\cdots+\left|\theta_{2}\right|
\end{aligned}
$$

$\|\tilde{x}\|_{\infty}=\max _{i}\left|x_{i}\right|$,

$$
\begin{aligned}
d^{*} & =(n+1)\|\mathbf{P}\| d^{2}\left(\sum_{i=1}^{n} \frac{1}{K^{i-1}}\right)^{2} \\
& =\frac{(n+1)\|\mathbf{P}\| d^{2}\left(\sum_{i=0}^{n-1}(i+1) K^{i}+\sum_{i=n}^{2 n-2}(2 n-i-1) K^{i}\right)}{K^{2 n-2}} .
\end{aligned}
$$

Here, this paper cleverly performs the extremum processing of the growth conditions and, through the normalization of the maximum and minimum results, converts the function growth conditions into uncertain parameter growth conditions.

3.2. Backstepping Method to Design an Output Feedback Controller. In this section, we will get the output feedback controller through the backstepping method. Based on the consideration of system simplification, the following coordinate transformations are introduced for stochastic nonlinear systems:

$$
\begin{aligned}
& w_{1}=\widehat{x}_{1}, \\
& w_{i}=\widehat{x}_{i}-\beta_{i-1}\left(\hat{x}_{1}, \ldots, \hat{x}_{i-1}\right) .
\end{aligned}
$$


In the new states, the idea of virtual control is introduced, that is, $\beta_{i-1}$ is the virtual controller to be designed.

Based on the observer system function $V_{0}(\widetilde{x})$ in Section 3.1, we construct a new set of Lyapunov functions:

$$
V_{1}\left(\tilde{x}, w_{1}\right)=V_{0}(\tilde{x})+\sqrt{\left|p_{1}(x)\right|+\cdots+\left|p_{n}(x)\right|} \frac{1}{\bar{\theta}+1} w_{1}^{\bar{\theta}+1}
$$

Using equations (8)-(15), one can obtain

$$
\begin{aligned}
L V_{1} \leq & -\left((n+1) n K-\sqrt{\left|p_{1}(x)\right|+\cdots+\left|p_{n}(x)\right|} d^{*}\left(\sum_{i=1}^{n} K^{(i-1) \bar{\theta}-(i-1)}\right)^{2}\right)\|\widetilde{x}\|_{\infty}^{2 p} \\
& +\sqrt{\left|p_{1}(x)\right|+\cdots+\left|p_{n}(x)\right|} d^{*}\left(\widehat{x}_{1}^{2 \bar{\theta}}+\frac{\widehat{x}_{2}^{2 \bar{\theta}}}{K^{2}}+\cdots+\frac{\widehat{x}_{n}^{2 \bar{\theta}}}{K^{2 n-2}}\right)+w_{1}^{\bar{\theta}}\left(\widehat{x}_{2}+K h_{1} \tilde{x}_{1}\right) \\
\leq & -\left((n+1) n K-\sqrt{\left|p_{1}(x)\right|+\cdots+\left|p_{n}(x)\right|} d^{*}\left(\sum_{i=1}^{n} K^{(i-1) \bar{\theta}-(i-1)}\right)^{2}\right)\|\widetilde{x}\|_{\infty}^{2 p} \\
& \left.+\sqrt{\left|p_{1}(x)\right|}+\cdots+\left|p_{n}(x)\right| d\left(\frac{\hat{x}_{3}^{2 \bar{\theta}}}{K^{4}}+\cdots+\frac{\hat{x}_{n}^{2 \bar{\theta}}}{K^{2 n-2}}\right)+\frac{\widehat{x}_{2}^{2 p}}{K^{2}}+w_{1}^{2 p}\right) \\
& +w_{1}^{\bar{\theta}} w_{2}+w_{1}^{\bar{\theta}} \beta_{1}+K \tilde{x}_{1}^{2}+\frac{K}{4} h_{1}^{2} w_{1}^{2 \bar{\theta}} \cdot
\end{aligned}
$$

In fact, in the iterative process of this paper, there is an idea of knowing the results before making assumptions. Therefore, we need to make certain necessary assumptions. These assumptions are not only constraints on the research object model but also our implementation. And this is a necessary condition in the controller derivation process. Due to the omission of the first draft, certain assumptions and constraints are actually required here.

Here, according to the backstepping theory, using (8)-(16) to select a specific relationship $K \geq 2^{2 p} n d^{*}$, one can get

$$
\begin{aligned}
& \sqrt{\left|p_{1}(x)\right|+\cdots+\left|p_{n}(x)\right|} \mathrm{d} w_{1}^{2} \leq \frac{K}{2} w_{1}^{2}, \\
& \frac{\hat{x}_{2}^{2 \bar{\theta}}}{K^{2}} \leq\left(\frac{1}{K^{2}} w_{2}^{2 \bar{\theta}}+\frac{1}{K^{2}} \beta_{1}^{2 \bar{\theta}}\right) .
\end{aligned}
$$

Therefore, after scaling down the inequality, we can get the first virtual controller as

$$
\begin{aligned}
& \beta_{1}=-K \sqrt{\left|p_{1}(x)\right|+\cdots+\left|p_{n}(x)\right|} v_{1} w_{1}^{\bar{\theta}}, \\
& v_{1}=\frac{1}{2}\left(1+\frac{h_{1}^{2}}{2}+n\right) .
\end{aligned}
$$

The idea of reverse thrust control needs to be explained here. Only in the continuous reverse thrust process, continuous virtual controllers will appear, and these virtual controllers have certain mutual laws. Only when the theory of virtual control is added in the amplification process of Lyapunov formulas of different orders can the final negative definite result be obtained, and the result of this item one puts forward is the result of applying virtual control. 
Importing the first virtual controller into the first Ito differential equation, a new Ito differential equation can be obtained:

$$
\begin{aligned}
& L V_{1} \leq-\left((n+1) n K-\sqrt{\left|p_{1}(x)\right|+\cdots+\left|p_{n}(x)\right|} d\left(\sum_{i=1}^{n} K^{(i-1) \bar{\theta}-(i-1)}\right)^{2}\right)\|\tilde{x}\|_{\infty}^{2 \bar{\theta}} \\
& +\sqrt{\left|p_{1}(x)\right|+\cdots+\left|p_{n}(x)\right|} d\left(\frac{\hat{x}_{3}^{2 \bar{\theta}}}{K^{4}}+\cdots+\frac{\hat{x}_{n}^{2 \bar{\theta}}}{K^{2 n-2}}+\frac{2}{K^{2}} w_{2}^{2 \bar{\theta}}\right)+w_{1}^{\bar{\theta}} w_{2}+w_{1}^{\bar{\theta}} \beta_{1}+K \widetilde{x}_{1}^{2}+\frac{K}{4} h_{1}^{2} w_{1}^{2 \bar{\theta}} \\
& \leq-\left(((n+1) n-1) K-\sqrt{\left|p_{1}(x)\right|+\cdots+\left|p_{n}(x)\right|} d\left(\sum_{i=1}^{n} K^{(i-1) \bar{\theta}-(i-1)}\right)^{2}\right)\|\tilde{x}\|_{\infty}^{2 \bar{\theta}} \\
& +\sqrt{\left|p_{1}(x)\right|+\cdots+\left|p_{n}(x)\right|} d\left(\frac{\hat{x}_{3}^{2 \bar{\theta}}}{K^{4}}+\cdots+\frac{\widehat{x}_{n}^{2 \bar{\theta}}}{K^{2 n-2}}+\frac{2}{K^{2}} w_{2}^{2 \bar{\theta}}\right) \\
& +w_{1}^{\bar{\theta}} w_{2}+w_{1}^{\bar{\theta}} \beta_{1}+K \sqrt{\left|p_{1}(x)\right|+\cdots+\left|p_{n}(x)\right|}\left(\frac{1}{2}+\frac{h_{1}^{2}}{4}\right) w_{1}^{2 \bar{\theta}} \\
& \leq-\left(((n+1) n-1) K-\sqrt{\left|p_{1}(x)\right|+\cdots+\left|p_{n}(x)\right|} d\left(\sum_{i=1}^{n} K^{(i-1) \bar{\theta}-(i-1)}\right)^{2}\right)\|\tilde{x}\|_{\infty}^{2 \bar{\theta}} \\
& +\sqrt{\left|p_{1}(x)\right|+\cdots+\left|p_{n}(x)\right|} d\left(\frac{\widehat{x}_{3}^{2 \bar{\theta}}}{K^{4}}+\cdots+\frac{\widehat{x}_{n}^{2 \bar{\theta}}}{K^{2 n-2}}+2 v_{1}^{2 p} \sqrt{\underbrace{w_{1}^{2 \theta} \cdot w_{1}^{2 \theta}}_{2 \bar{\theta}}}+\frac{2}{K^{2}} w_{2}^{2 \bar{\theta}}\right)-n K w_{1}^{2 \bar{\theta}} \\
& \leq-\left(((n+1) n-1) K-\sqrt{\left|p_{1}(x)\right|+\cdots+\left|p_{n}(x)\right|} d\left(\sum_{i=1}^{n} K^{(i-1) \bar{\theta}-(i-1)}\right)^{2}\right)\|\widetilde{x}\|_{\infty}^{2 \bar{\theta}} \\
& +\sqrt{\left|p_{1}(x)\right|+\cdots+\left|p_{n}(x)\right|} d\left(\frac{\hat{x}_{3}^{2 p}}{K^{4}}+\cdots+\frac{\hat{x}_{n}^{2 p}}{K^{2 n-2}}+\bar{\theta} n d^{*} v_{1}^{2 \bar{\theta}}\right)-K w_{1}^{2 p} .
\end{aligned}
$$

After obtaining the first virtual controller, through the theory of coherent analogy, we can infer backwards in turn until we get the $i-1$ virtual controller.

$$
\beta_{1}\left(\hat{x}_{1}\right), \ldots, \beta_{i-1}\left(\hat{x}_{1}, \ldots \widehat{x}_{i-1}\right),
$$

$$
\begin{gathered}
\beta_{1}\left(\hat{x}_{1}\right)=-K \sqrt{\left|p_{1}(x)\right|+\cdots+\left|p_{n}(x)\right|} v_{1} w_{1}^{\bar{\theta}} \\
\vdots \\
\beta_{i-1}\left(\widehat{x}_{1}, \cdots \widehat{x}_{i-1}\right)=-K^{i-1} \sqrt{\left|p_{1}(x)\right|+\cdots+\left|p_{n}(x)\right|} v_{i-1} w_{i-1}^{\bar{\theta}},
\end{gathered}
$$


where

$$
\begin{gathered}
v_{1}=\frac{1}{2}+\frac{h_{1}^{2}}{4}+n \\
\vdots \\
v_{i-1}=\frac{1}{4}\left(h_{i-1}+v_{i-2} h_{i-2}+v_{i-2} v_{i-3} h_{i-3}+\cdots+v_{i-2} v_{i-3} \cdots v_{1} h_{1}\right)^{2}+\cdots+\frac{1}{4}\left(v_{i-3} v_{i-2}-v_{i-2}^{2}\right)^{2}+v_{i-2}+1+n-(i-2) .
\end{gathered}
$$

The constant $v_{j}>0$ is a real constant that does not depend on $K$ so that the expression of using the Lyapunov function for the $i$ time is

$$
V_{i-1}\left(\tilde{x}, w_{1}, \ldots, w_{i-1}\right)=V_{0}(\tilde{x})+\sqrt{\left|p_{1}(x)\right|+\cdots+\left|p_{n}(x)\right|} \frac{1}{\bar{\theta}+1} \sum_{j=1}^{i-1} \frac{1}{K^{j}} w_{j}^{\bar{\theta}+1} .
$$

This Lyapunov function is satisfying

$$
\begin{aligned}
L V_{i-1} \leq & -\left(((n+1) n-(i-1)) K-\sqrt{\left|p_{1}(x)\right|+\cdots+\left|p_{n}(x)\right|} d\left(\sum_{i=1}^{n} K^{(i-1) \bar{\theta}-(i-1)}\right)^{2}\right)\|\tilde{x}\|_{\infty}^{2 \bar{\theta}} \\
& +\sqrt{\left|p_{1}(x)\right|+\cdots+\left|p_{n}(x)\right|} d\left(\frac{\hat{x}_{i+1}^{2 \bar{\theta}}}{\left.K^{2 i}+\cdots+\frac{\widehat{x}_{n}^{2 \bar{\theta}}}{K^{2 n-2}}\right)}\right. \\
& -\sum_{j=1}^{i-1} \frac{1}{K^{2 j-2}}\left(\left(n-4^{(\bar{\theta}-1)(j-1)}\right) K-\sqrt{\left|p_{1}(x)\right|+\cdots+\left|p_{n}(x)\right|} \bar{\theta} d v_{j}^{2 p}\right) w_{j}^{2 \bar{\theta}} \\
& +\sqrt{\left|p_{1}(x)\right|+\cdots+\left|p_{n}(x)\right|} d \frac{2}{K^{2(i-1)}} w_{i}^{2 \bar{\theta}}+\frac{1}{K^{2(i-2)}} w_{i-1}^{\bar{\theta}} w_{i} .
\end{aligned}
$$

The next Lyapunov function of the recursive series is $V_{i}\left(\widetilde{x}, w_{1}, \ldots, w_{i-1}\right)=V_{i-1}\left(\widetilde{x}, w_{1}, \ldots, w_{i-1}\right)$

$$
+\sqrt{\left|p_{1}(x)\right|+\cdots+\left|p_{n}(x)\right|} \frac{1}{\bar{\theta}+1} \cdot \frac{1}{K^{i}} w_{i}^{\bar{\theta}+1} .
$$

Whether the system's Ito differential equation based on the latest Lyapunov function satisfies negative definiteness needs to be obtained by the following proof. Using $K \geq \sqrt{\left|p_{1}(x)\right|+\cdots+\left|p_{n}(x)\right|} d$ and combining equations (18)-(25), one can get 


$$
\begin{aligned}
& L V_{i} \leq-\left(((n+1) n-(i-1)) K-\sqrt{\left|p_{1}(x)\right|+\cdots+\left|p_{n}(x)\right|} d\left(\sum_{i=1}^{n} K^{(i-1) p-(i-1)}\right)^{2}\right)\|\tilde{x}\|_{\infty}^{2 \bar{\theta}} \\
& +\sqrt{\left|p_{1}(x)\right|+\cdots+\left|p_{n}(x)\right|} d\left(\frac{\widehat{x}_{i+2}^{2 \bar{\theta}}}{K^{2(i+1)}}+\cdots+\frac{\hat{x}_{n}^{2 \bar{\theta}}}{K^{2 n-2}}\right) \\
& -\sum_{j=1}^{i-1} \frac{1}{K^{2 j-2}}\left(\left(n-4^{(\theta-1)(j-1)}\right) K-2^{4 \theta-1} p n d^{*} v_{j}^{2 \theta}\right) w_{j}^{2 \theta} \\
& +\sqrt{\left|p_{1}(x)\right|+\cdots+\left|p_{n}(x)\right|} d \frac{1}{K^{2 i}} \widehat{x}_{i+1}^{2 \bar{\theta}}+\frac{1}{K^{2(i-1)}} w_{i}^{2 \bar{\theta}} \widehat{x}_{i+1}+\frac{1}{K^{i}} w_{i}^{\bar{\theta}} \widehat{x}_{i+1} \\
& +w_{i}^{\bar{\theta}} h_{i} \tilde{x}_{i}+\frac{1}{K^{i-1}} w_{i}^{\bar{\theta}} v_{i-1} p w_{i-1}^{\bar{\theta}-1}\left(\widehat{x}_{i}+K^{i-1} h_{i-1} \tilde{x}_{1}\right) \\
& +\frac{1}{K^{i-2}} w_{i}^{\bar{\theta}} v_{i-1} v_{i-2} p v_{i-2}^{\bar{\theta}-1}\left(\widehat{x}_{i-1}+K^{i-2} h_{i-2} \widetilde{x}_{1}\right)+\cdots+\frac{1}{K^{i-(i-1)}} w_{i}^{\bar{\theta}} v_{i-1} v_{i-2} \cdot v_{1} p w_{1}^{\bar{\theta}-1}\left(\widehat{x}_{2}+K h_{1} \widetilde{x}_{1}\right) \\
& \leq-\left(((n+1) n-i) K-\sqrt{\left|p_{1}(x)\right|+\cdots+\left|p_{n}(x)\right|} d\left(\sum_{i=1}^{n} K^{(i-1) \bar{\theta}-(i-1)}\right)^{2}\right)\|\widetilde{x}\|_{\infty}^{2 \bar{\theta}} \\
& +\sqrt{\left|p_{1}(x)\right|+\cdots+\left|p_{n}(x)\right|} d\left(\frac{\widehat{x}_{i+2}^{2 \bar{\theta}}}{K^{2(i+1)}}+\cdots+\frac{\widehat{x}_{n}^{2 \bar{\theta}}}{K^{2 n-2}}\right) \\
& -\sum_{j=1}^{i} \frac{1}{K^{2 j-2}}\left(\left(n-4^{(\bar{\theta}-1) j}\right) K-p \sqrt{\left|p_{1}(x)\right|+\cdots+\left|p_{n}(x)\right|} d^{*} v_{j}^{2 \bar{\theta}}\right) w_{j}^{2 \bar{\theta}} \\
& +\sqrt{\left|p_{1}(x)\right|+\cdots+\left|p_{n}(x)\right|} d \frac{2}{K^{2 i}} w_{i+1}^{2 \bar{\theta}} \\
& +\sqrt{\left|p_{1}(x)\right|+\cdots+\left|p_{n}(x)\right|} d \frac{2}{K^{2 i}} \beta_{i}^{2 \bar{\theta}}+\frac{1}{K^{2(i-1)}} w_{i}^{\bar{\theta}} w_{i+1} \\
& +\frac{1}{K^{2(i-1)}} w_{i}^{\bar{\theta}} \beta_{i}+\frac{1}{4 K^{2 i-3}} w_{i}^{2 \bar{\theta}}\left(h_{i}+v_{i-1} h_{i-1} \cdots+v_{i-1} v_{i-2} \cdot v_{1} h_{1}\right)^{2} \\
& +\cdots+\frac{1}{4 K^{2 i-3}} w_{i}^{2 \bar{\theta}}\left(v_{i-3} v_{i-2}-v_{i-2}^{2}\right)^{2}+\frac{1}{K^{2 i-3}} w_{i}^{2 \bar{\theta}}\left(v_{i-1}+1\right)
\end{aligned}
$$

The system is differentiated by Ito microdifferentiation and needs to satisfy the following results: 


$$
\begin{aligned}
L V_{i} \leq & -\left(((n+1) n-i) K-\sqrt{\left|p_{1}(x)\right|+\cdots+\left|p_{n}(x)\right|} d^{*}\left(\sum_{i=1}^{n} K^{(i-1) \bar{\theta}-(i-1)}\right)^{2}\right)\|\tilde{x}\|_{\infty}^{2 \bar{\theta}} \\
& +\sqrt{\left|p_{1}(x)\right|+\cdots+\left|p_{n}(x)\right|} d^{*}\left(\frac{\widehat{x}_{i+2}^{2 \bar{\theta}}}{K^{2(i+1)}}+\cdots+\frac{\widehat{x}_{n}^{2 \bar{\theta}}}{K^{2 n-2}}\right) \\
& -\sum_{j=1}^{i} \frac{1}{K^{2 j-2}}\left(\left(n-44^{(\bar{\theta}-1) j}\right) K-\sqrt{\left|p_{1}(x)\right|+\cdots+\left|p_{n}(x)\right|} \bar{\theta} d^{*} v_{j}^{2 \bar{\theta}}\right) w_{j}^{2 \bar{\theta}} \\
& +\sqrt{\left|p_{1}(x)\right|+\cdots+\left|p_{n}(x)\right|} d^{*} \frac{2}{K^{2 i}} w_{i+1}^{2 \bar{\theta}}+\frac{1}{K^{2(i-1)}} w_{i}^{\bar{\theta}} w_{i+1} \cdot
\end{aligned}
$$

By selecting the $i$ virtual controller,

$$
\begin{aligned}
\beta_{i}\left(\widehat{x}_{1}, \ldots, \hat{x}_{i}\right)= & -K^{i} \sqrt{\left|p_{1}(x)\right|+\cdots+\left|p_{n}(x)\right|} v_{i} w_{i}^{\bar{\theta}}, \\
v_{i}= & h_{i}+v_{i-1} h_{i-1}+v_{i-1} v_{i-2} h_{i-2}+\cdots \\
& +v_{i-1} v_{i-2} \ldots v_{1} h_{1} .
\end{aligned}
$$

Next, repeating the recursive process of step $i$, one can get

$$
\begin{aligned}
L V_{n-1} \leq & -\left(((n+1) n-(n-1)) K-\sqrt{\left|p_{1}(x)\right|+\cdots+\left|p_{n}(x)\right|} d^{*}\left(\sum_{i=1}^{n} K^{(i-1) \bar{\theta}-(i-1)}\right)^{2}\right)\|\tilde{x}\|_{\infty}^{2 \bar{\theta}} \\
& -\sum_{j=1}^{n-1} \frac{1}{K^{2 j-2}}\left(\left(n-4^{(\bar{\theta}-1)(j-1)}\right) K-\sqrt{\left|p_{1}(x)\right|+\cdots+\left|p_{n}(x)\right|} \bar{\theta}^{*} v_{j}^{2 \bar{\theta}}\right) w_{j}^{2 \bar{\theta}} \\
& +\sqrt{\left|p_{1}(x)\right|+\cdots+\left|p_{n}(x)\right|} d \frac{2}{K^{2(n-1)}} w_{n}^{2 \bar{\theta}}+\frac{1}{K^{2(n-2)}} w_{n-1}^{\bar{\theta}} w_{n} .
\end{aligned}
$$

Here, the Lyapunov function is chosen as

$$
\begin{aligned}
& \begin{aligned}
& V_{n-1}\left(\tilde{x}, w_{1}, \ldots, w_{n-1}\right)= V_{n-2}\left(\tilde{x}, w_{1}, \ldots, w_{n-2}\right) \\
&+\sqrt{\left|p_{1}(x)\right|+\cdots+\left|p_{n}(x)\right|} \frac{1}{K^{n-1}} \cdot \frac{1}{\bar{\theta}+1} w_{n}^{2 \bar{\theta}} . \\
& u\left(\hat{x}_{[n]}\right)=-K^{n} \sqrt{\left|p_{1}(x)\right|+\cdots+\left|p_{n}(x)\right|} v_{n} w_{n}^{\bar{\theta}} .
\end{aligned}
\end{aligned}
$$
controller

Use the last Lyapunov function

$$
\begin{aligned}
V_{n}\left(\tilde{x}, w_{1}, \ldots, w_{n}\right)= & (n+1) \tilde{x}^{T} P \widetilde{x} \\
& +\sqrt{\left|p_{1}(x)\right|+\cdots+\left|p_{n}(x)\right|} \frac{1}{\bar{\theta}+1} \sum_{j=1}^{n} \frac{1}{K^{j}} w_{j}^{\bar{\theta}+1} .
\end{aligned}
$$


One can get the last Ito system function:

$$
\begin{aligned}
L V_{n} \leq & -\left(n^{2} K-\sqrt{\left|p_{1}(x)\right|+\cdots+\left|p_{n}(x)\right|} d\left(\sum_{i=1}^{n} K^{(i-1) \bar{\theta}-(i-1)}\right)^{2}\right)\|\tilde{x}\|_{\infty}^{2 \bar{\theta}} \\
& -\sum_{j=1}^{n-1} \frac{1}{K^{2 j-2}}\left(\left(n-4^{(\bar{\theta}-1)(j-1)}\right) K-\sqrt{\left|p_{1}(x)\right|+\cdots+\left|p_{n}(x)\right|} \bar{\theta} d v_{j}^{2 \bar{\theta}}\right) w_{j}^{2 \bar{\theta}}-\frac{1}{K^{n}} w_{n}^{2 \bar{\theta}} .
\end{aligned}
$$

That is, the final output feedback controller is controlled by a high-gain term $K^{n} \sqrt{\left|p_{1}(x)\right|+\cdots+\left|p_{n}(x)\right|}$.

\section{System Stability Analysis}

The stability analysis of the closed-loop system is performed below, and the main conclusions of this paper are obtained. First, according to system (3) and (28)-(31), one needs to determine the size relationship between

$$
\begin{aligned}
& \left|\sqrt{\left|p_{1}(x)\right|+\cdots+\left|p_{n}(x)\right|} \frac{\bar{\theta} \mathrm{d} v_{n}^{2 \bar{\theta}}}{n-4^{(\bar{\theta}-1)(n-1)} \mid}\right|, \\
& K^{n} \sqrt{\left|p_{1}(x)\right|+\cdots+\left|p_{n}(x)\right|} .
\end{aligned}
$$

Substituting equation (34) into equation (33), it can get the following inequality:

$$
\begin{aligned}
K> & \sqrt{\left|p_{1}(x)\right|+\cdots+\left|p_{n}(x)\right|} \cdot \frac{\bar{\theta} v_{n}^{2 \bar{\theta}}}{n-4^{(\bar{\theta}-1)(n-1)}} \cdot \frac{(n+1)\|P\| d^{2}}{K^{2 n-2}} \\
& \cdot\left(\sum_{i=0}^{n-1}(i+1) K^{i}+\sum_{i=n}^{2 n-2}(2 n-i-1) K^{i}\right) .
\end{aligned}
$$

The above formula is equivalent to

$$
\begin{aligned}
K^{2 n-1}> & \frac{n}{n-4^{(\bar{\theta}-1)(n-1)}} \cdot \sqrt{\left|p_{1}(x)\right|+\cdots+\left|p_{n}(x)\right|} \cdot \bar{\theta} \cdot v_{n}^{2 \bar{\theta}} \\
& \cdot(n+1)\|P\| d^{2}\left(\sum_{i=0}^{n-1}(i+1) K^{i}+\sum_{i=n}^{2 n-2}(2 n-i-1) K^{i}\right) .
\end{aligned}
$$

According to the factorization theorem of the real coefficient polynomial, (36) can be further expressed as

$$
\begin{aligned}
& \left(K-K_{1}\right)^{m_{1}} \ldots\left(K-K_{r}\right)^{m_{r}} \\
& \quad \cdot\left(K^{2}+c_{1} K+b_{1}\right)^{n_{1}} \ldots\left(K^{2}+c_{s} K+b_{s}\right)^{n_{s}}>0,
\end{aligned}
$$

where $m_{i}$ and $n_{j}$ are positive integers. It can be concluded that if there is at least one positive number for $K_{1}, \ldots, K_{r}$ under the condition of the appropriate value of $\bar{\theta}$, one can choose $K^{*}=\max _{1 \leq i \leq r}\left\{K_{i}\right\}$. Thus, there always exists $K^{*} \geq 0$ so that the closed-loop stochastic nonlinear system is globally asymptotically stable in the probability sense. Through the above analysis, one can get the following theorem.

Theorem 1. For stochastic nonlinear system (3) that satisfies function condition Assumption 1, there is a high-gain parameter $K^{*} \geq 0$ so that output feedback controller (31) designed in Section 3 of this paper can guarantee that, for any system initial value, closed-loop stochastic nonlinear system (3) exists almost everywhere and is unique; and for any system initial value, the closed-loop stochastic nonlinear system is globally asymptotically stable in the probability sense.

On the basis of Theorem 1, an inverse optimal controller is designed for system (3) so that it meets specific performance indicators while achieving the control objectives. First, Lemma 1 is given [37].

Lemma 1. Define the following controller:

$$
u=\alpha(x)=-R_{2}^{-1}\left(L_{\phi_{2}} V\right)^{T} \frac{\ell \gamma\left(\left|L_{\phi_{2}} V R_{2}^{-1 / 2}\right|\right)}{\left|L_{\phi_{2}} V R_{2}^{-1 / 2}\right|^{2}}
$$

where $\gamma(\cdot)$ is a class $K_{\infty}$ function, $\ell \gamma(s)=s(\dot{\gamma})^{-1}(s)-\gamma\left((\dot{\gamma})^{-1}(s)\right)$, and $R_{2}(x)$ is a matrix value function and satisfies $R_{2}(x)=R_{2}^{T}(x)>0$. It can be known from Theorem 1 that output feedback controller (31) guarantees that system (3) is globally asymptotically stable in the sense of probability, and then the following controller is constructed:

$$
u^{*}=\beta^{*}(x)=-\frac{\theta}{2} R_{2}^{-1}\left(L_{\phi_{2}} V\right)^{T} \frac{\ell \gamma\left(\left|L_{\phi_{2}} V R_{2}^{-1 / 2}\right|\right)}{\left|L_{\phi_{2}} V R_{2}^{-1 / 2}\right|^{2}}, \quad \theta \geq 2,
$$

which can minimize the performance function

$$
J(u)=E\left(\int_{0}^{\infty}\left(l(x)+\theta^{2} \gamma\left(\frac{2}{\theta}\left|R_{2}(x)^{1 / 2} u\right|\right)\right) \mathrm{d} \tau\right),
$$

to solve the inverse optimal stabilization problem of stochastic nonlinear systems (3). $l(x)$ satisfies the following equation: 


$$
\begin{aligned}
l(x)= & 2 \theta\left(\operatorname{e\gamma }\left(\left|L_{\phi_{2}} V R_{2}^{-1 / 2}\right|\right)-\frac{1}{2} \operatorname{Tr}\left(\phi_{1}^{T} \frac{\partial^{2} V}{\partial x^{2}} \phi_{1}\right)\right) \\
& +\theta(\theta-2) \ell \gamma\left(\left|L_{\phi_{2}} V R_{2}^{-1 / 2}\right|\right) .
\end{aligned}
$$

Next, the design process of the inverse optimal controller of the system studied in this paper is given. First, the differential equation between the state error and the state estimation is given:

$$
\left(\begin{array}{l}
\mathrm{d} \tilde{x} \\
\mathrm{~d} \widehat{x}
\end{array}\right)=\bar{\phi}_{1}(\widetilde{x}, \widehat{x}) \mathrm{d} \omega+\bar{\phi}_{2}(\widetilde{x}, \widehat{x}) u \mathrm{~d} t
$$

Selecting

$$
\gamma(s)=\sqrt{\left|p_{1}(x)\right|+\cdots+\left|p_{n}(x)\right|} \frac{1}{2 K^{2(n-1)}} s^{2},
$$

one can obtain $\left(\gamma^{\prime}\right)^{-1}(s)=K^{2(n-1)} s$. Combining Lemma 1, one obtains

$$
u=\beta(\widehat{x})=-R_{2}^{-1}(\widehat{x}) \sqrt{\left|p_{1}(x)\right|+\cdots+\left|p_{n}(x)\right|} \frac{1}{K^{2(n-1)}} w_{n}^{\bar{\theta}} \frac{1}{2} K^{2(n-1)},
$$

where defined $R_{2}(\hat{x}) \cdot 2 K^{n} v_{n}=1$. The inverse optimal controller can be obtained as

$$
u^{*}=\beta^{*}(\widehat{x})=-\frac{\bar{\theta}}{2} R_{2}^{-1}(\widehat{x}) \frac{1}{K^{2(n-1)}} w_{n}^{\bar{\theta}} K^{2(n-1)}=-\frac{\bar{\theta}}{2} R_{2}^{-1}(\widehat{x}) w_{n}^{\bar{\theta}}
$$

Through the above analysis, the theorem of the inverse optimal controller is obtained.

Theorem 2. The following controller

$$
u^{*}=-\mu \sqrt{\left|p_{1}(x)\right|+\cdots+\left|p_{n}(x)\right|} K^{n} v_{n} w_{n}^{\bar{\theta}}
$$

exists so that stochastic nonlinear system (3) can perform inverse optimal stabilization design in the sense of probability and minimize the following target performance function:

$$
J(u)=E\left(\int_{0}^{\infty}\left(l(\tilde{x}, \widehat{x})+\frac{1}{K^{2(n-1)}} \frac{v_{n}^{-1}(\widehat{x})}{K^{n}} u^{2}\right) \mathrm{d} r\right) .
$$

\section{Simulation Example of Subsea Electroexecution Robot System}

The underwater subsea intelligent electroexecution robot composite position control system is used in practical offshore engineering such as oil and gas extraction and production, especially in the field of subsea oil and gas development. In practical engineering applications, this system presents uncertain and nonlinear characteristics. The electrohydraulic composite valve position control system is composed of the water equipment part and underwater equipment part (as shown in Figure 1), where the water part includes power supply unit, hydraulic station, and other power units and other control units; the underwater part includes electromagnetic reversing valve, hydraulic actuator, and electronic control unit controlling the electromagnetic reversing valve. The electronic control unit is internally designed for the controller logic. In actual marine engineering applications, in order to ensure the stability, reliability, and safety of the system, the response speed of the valve position control needs to be stable, accurate, and timely; during the valve position opening/closing process, it is also subject to the hydraulic power unit from the surface. The system is simultaneously subjected to hydraulic driving force, spring restoring force, and the force generated by the fluid medium inside the oil and gas pipeline during the opening of the actuator.

Combining existing research results $[38,39]$, the model of the electrohydraulic composite valve position control system during the valve position opening process is obtained as follows:

$$
\begin{aligned}
p\left(S-S_{1}\right)+\rho g H S_{1} & =m \frac{\mathrm{d}^{2} \tau}{\mathrm{d} t^{2}}+\left(\rho g H+\frac{128 \mu l_{p} Q}{\pi d_{p}^{4}}\right)\left(S-S_{2}\right)+k\left(L_{0}+\tau\right)+f\left(\tau, \frac{\mathrm{d} \tau}{\mathrm{d} t}\right)+w(t) \\
Q_{h} & =v\left(S-S_{1}\right)\left(2 * \frac{\left(P_{1}-P_{2}\right)}{\rho_{h}}\right) .
\end{aligned}
$$

Due to the pressure instability of the oil and gas medium and the formation of gravel and other components, it will cause some interference during the opening of the electrohydraulic composite valve position. Therefore, there are interference factors in the pipeline of
Figure 2. At the same time, due to factors such as the change in the viscosity coefficient of the control fluid with temperature and the presence of vibrations in the hydraulic system, the resistance during the valve opening process has the characteristics of meeting the growth 


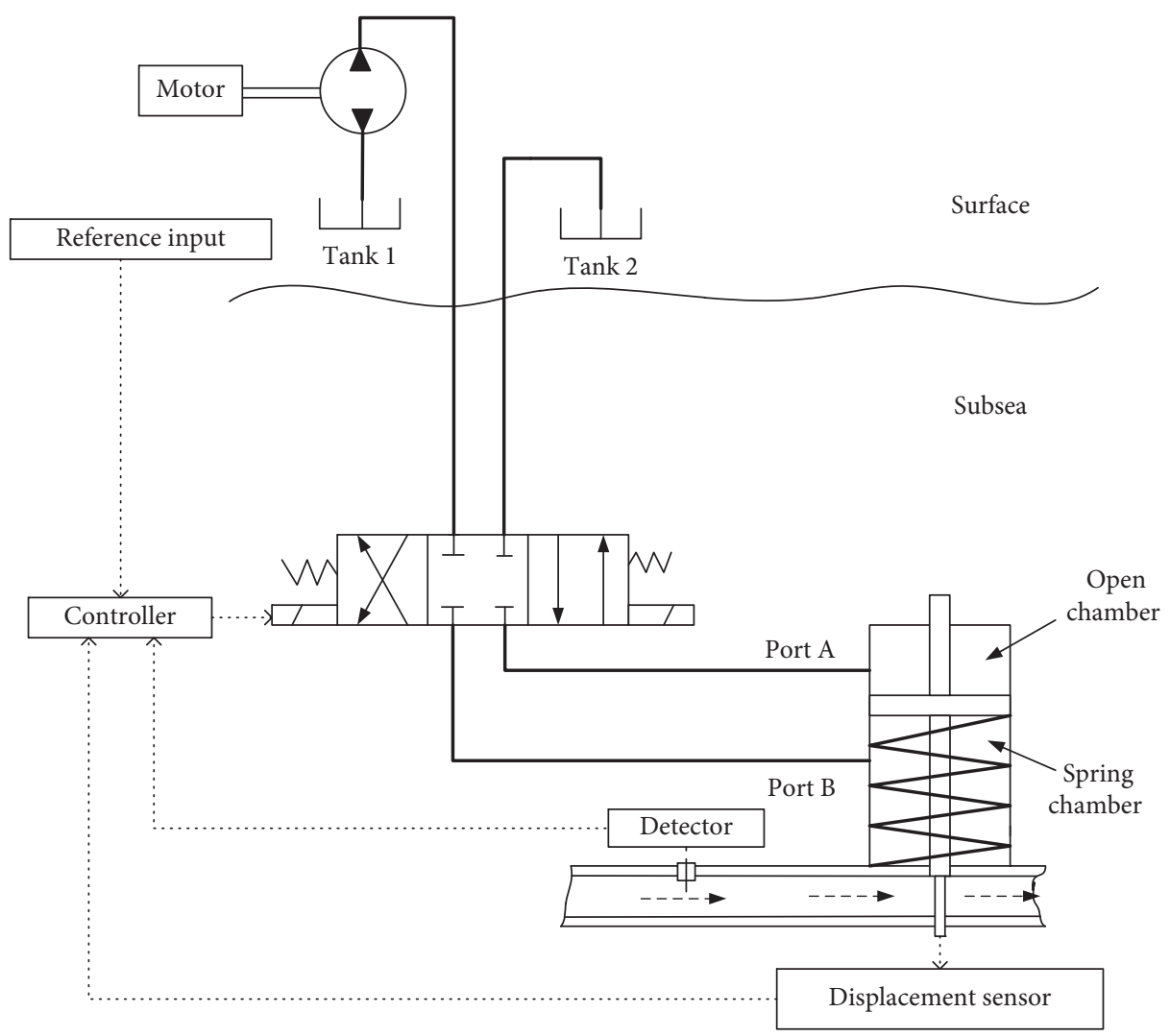

Figure 1: Underwater electrohydraulic composite valve position control system.

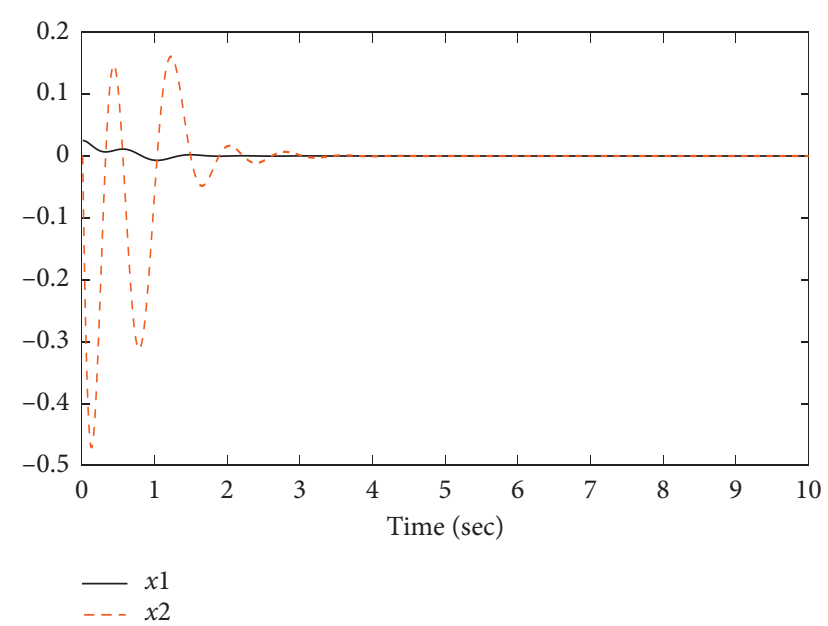

FIGURE 2: The trajectory of system states.

conditions of functional constraints. Therefore, the model of the underwater valve matches the stochastic nonlinear system we studied above.

Next, we use the observer and controller designed in this paper to control the electrohydraulic valve position and use computer simulation software for simulation analysis and verification. Through the analysis of the electrohydraulic composite valve position control system, the following state variables, measurement outputs, and control output variables are defined:

$$
\begin{aligned}
x_{1} & =\tau, \\
x_{2} & =\frac{\mathrm{d} \tau}{\mathrm{d} t}, \\
\mathrm{~d} x_{1} & =x_{2} \mathrm{~d} t+\mathrm{d} x_{1}^{p(x)} \sin x_{2} \mathrm{~d} \omega, \\
\mathrm{d} x_{2} & =u \mathrm{~d} t+d\left(x_{1}^{p(x)}+x_{2}^{(x)}\right) \mathrm{d} \omega, \\
y & =x_{1} .
\end{aligned}
$$

According to the definition of the state variable in the above formula, in actual application, on the one hand, the valve displacement can be measured, but the measurement of the valve displacement speed is difficult, which will directly affect the design of the state feedback controller; on the other hand, in actual application, the valve displacement sensor has a certain transmission delay and packet loss, which also has a certain impact on the design effect of the controller. Combining the measurement output with the "control fluid inlet pressure," since the monitoring and collection of the "control fluid inlet pressure" are located inside the control module in actual offshore oil and gas production, it has stable measurement and fast transmission effects, so it is established by combining this measurement. Observers and controllers are analyzed using the stability theorem and controller design method proposed in Section 3.sec3

Here, select $p(x)=\sin x$ where control $d=0.5$ which satisfies the assumptions of Section 2, and select the higherorder gain observer as 


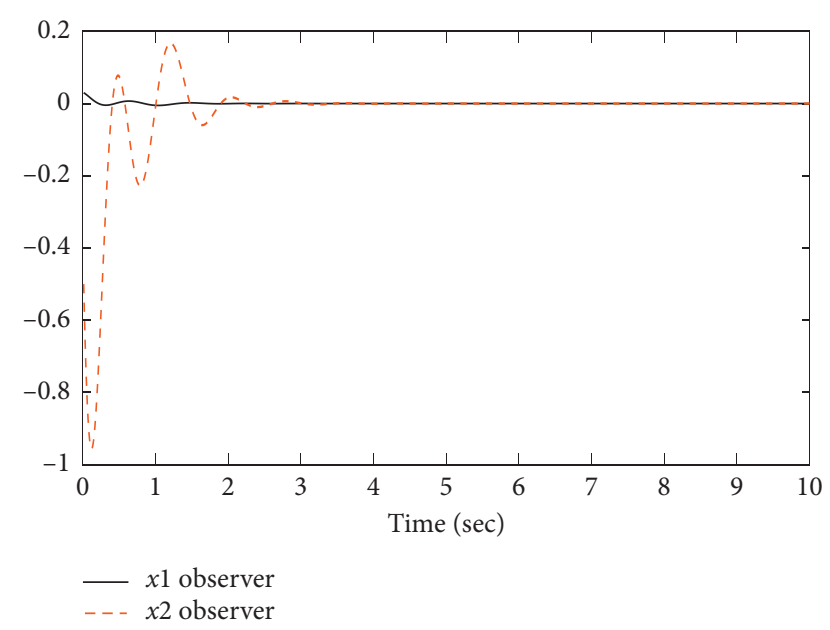

FIgURE 3: The trajectory of system observer states.

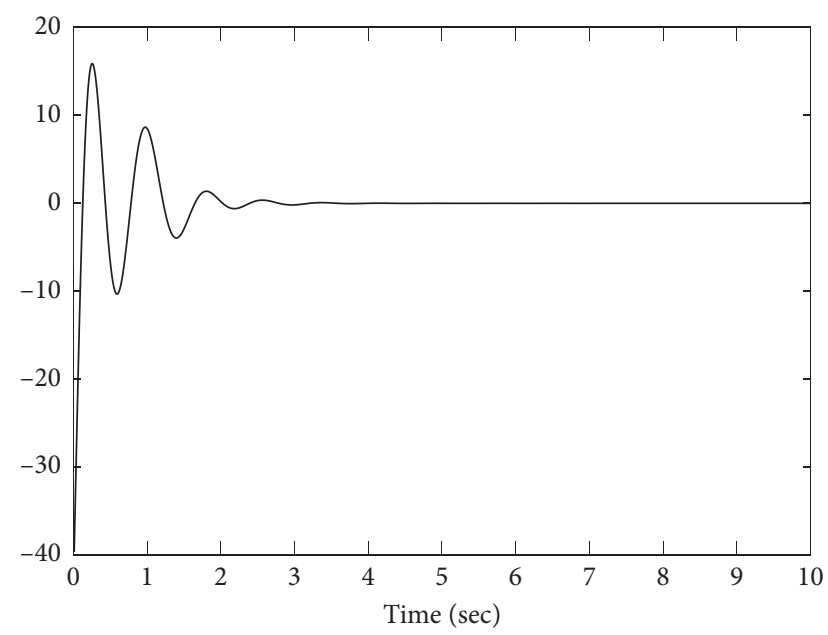

Figure 4: The trajectory of the output feedback controller.

$$
\begin{aligned}
& \dot{\hat{x}}_{1}=\widehat{x}_{2}+K\left(y-\widehat{x}_{1}\right), \\
& \dot{\hat{x}}_{2}=u+K^{2}\left(y-\widehat{x}_{1}\right) .
\end{aligned}
$$

Using the theory of the third controller design, after recursive operation,

$$
\begin{aligned}
& d^{*}=\frac{13}{4}\|\bar{\theta}\|\left(1+\frac{1}{K}\right)^{2}, \quad\|\bar{\theta}\|=20.5, K>65.8, \\
& u^{*}=-\|\bar{\theta}\| R_{2}^{-1}(\widehat{x}) w_{n}^{p}=-20.5 K^{2} v_{2} w_{2}^{3} .
\end{aligned}
$$

Furthermore, the parameters of the output feedback tracking controller are set according to Theorems 1 and 2 . Then, the system simulation is started, and the initial value is selected. Figures 3 and 4 show the response curve of closed-loop systems (49) and (50), which can be obtained by observing the simulation diagram. The actual and estimated states of two-dimensional systems (49) and (50) are bounded, and it gradually converges to zero. At the same time, the trajectories of the output feedback controller are also given. The choice of different values and the choice of different initial values will lead to a stable time and control amount. There are significant differences. Simulation results verify the effectiveness of the control scheme.

\section{Conclusion}

In this paper, for the first time, the output feedback controller is designed for a class of stochastic nonlinear systems that meet the growth conditions of uncertain functions. The multivariate function growth conditions we have assumed here have greatly relaxed the restrictions on the drift and diffusion terms in the original stochastic nonlinear system, making the system under study more general, and the scope of the application of the output feedback controller is also large. Here, we cleverly handle the problem of uncertain functions in the scaling process through the function maxima theory so that the Ito differential system can achieve output stabilization through Lyapunov function design so that the solution of stochastic nonlinear system objects satisfies the existence of uniqueness, ensuring that the system is globally asymptotically stable in the sense of probability, and it is further concluded that the system is inversely optimally stable in the sense of probability. Finally, we apply the theoretical results to the actual awake electrohydraulic valve control system and obtain good results.

There are some problems to be investigated: the systems studied in this theory and the paper are all continuous systems, so the study of control problems for the corresponding discrete systems is a future research direction. By querying the relevant literature, Wang Zidong's team, in the field of control theory, has done a lot of research work on the filtering and control of complex systems under the condition of missing information. Among them, the research on discrete systems has a greater impact on our subsequent research work.

\section{Data Availability}

All the data generated or analyzed during this research are included within this paper.

\section{Conflicts of Interest}

The authors declare that there are no conflicts of interest regarding the publication of this paper.

\section{Acknowledgments}

This work was supported by the National Key R\&D Program of China under Grant 2017YFB1301300, National Natural Science Foundation of China under Grant 61807010, Key R\&D Program of Zhejiang Province under Grant 2021C03013, Zhejiang Provincial Natural Science Foundation of China under Grant LZ21E050002, and Fundamental Research Funds for Provincial Universities of Zhejiang under Grant GK199900299012-026. 


\section{References}

[1] Y. Yu, G. Lu, C. Sun, and H. Liu, "Robust backstepping decentralized tracking control for a 3-DOF helicopter," Nonlinear Dynamics, vol. 82, no. 1-2, pp. 947-960, 2015.

[2] S.-H. Yoon, Y.-D. Kim, and S.-H. Park, "Constrained adaptive backstepping controller design for aircraft landing in wind disturbance and actuator stuck," International Journal of Aeronautical and Space Sciences, vol. 13, no. 1, pp. 74-89, 2012.

[3] J. M. Coron and L. Praly, "Adding an integrator for the stabilization problem," Systems \& Control Letters, vol. 17, no. 2, pp. 89-104, 1994.

[4] Z.-P. Jiang, A. R. Teel, and L. Praly, "Small-gain theorem for ISS systems and applications," Mathematics of Control, Signals, and Systems, vol. 7, no. 2, pp. 95-120, 1994.

[5] C. J. Qian and W. Lin, "Output feedback control of a class of nonlinear systems: a nonseparation principle paradigm," IEEE Transactions on Automatic Control, vol. 47, no. 10, pp. 1710-1715, 2002.

[6] H. Lei, J. F. Wei, and W. Lin, "A global observer for observable autonomous systems with bounded solution trajectories," in Proceeding of the 44th IEEE Conference on Decision and Control, pp. 1911-1916, Seville, Spain, December 2005.

[7] B. Yang and W. Lin, "Robust output feedback stabilization of uncertain nonlinear systems with uncontrollable and unobservable linearization," IEEE Transactions on Automatic Control, vol. 50, no. 5, pp. 619-630, 2005.

[8] Y. Chang, S. Zhang, N. D. Alotaibi, and A. F. Alkhateeb, "Observer-based adaptive finite-time tracking control for a class of switched nonlinear systems with unmodeled dynamics," IEEE Access, vol. 8, pp. 204782-204790, 2020.

[9] Y. Wang, N. Xu, Y. Liu, and X. Zhao, “Adaptive fault-tolerant control for switched nonlinear systems based on command filter technique," Applied Mathematics and Computation, vol. 392, p. 125725, 2021.

[10] Y. Wang, B. Niu, H. Wang, N. Alotaibi, and E. Abozinadah, "Neural network-based adaptive tracking control for switched nonlinear systems with prescribed performance: an average dwell time switching approach," Neurocomputing, vol. 435, pp. 295-306, 2021.

[11] M. KrstićI and I. Kanellakopoulos, "Adaptive nonlinear control without overparametrization," Systems \& Control Letters, vol. 19, no. 3, pp. 177-185, 1992.

[12] B. Wu, X.-H. Chang, and X. Zhao, "Fuzzy Ho output feedback control for nonlinear NCSs with quantization and stochastic communication protocol," IEEE Transactions on Fuzzy Systems, p. 1, 2020.

[13] H. Lei and W. Lin, "Universal adaptive control of nonlinear systems with unknown growth rate by output feedback," Automatica, vol. 42, no. 10, pp. 1783-1789, 2006.

[14] X.-H. Chang, Y. Liu, and M. Shen, "Resilient control design for lateral motion regulation of intelligent vehicle," IEEE/ ASME Transactions on Mechatronics, vol. 24, no. 6, pp. 2488-2497, 2019.

[15] S. S. Pavlichkov, S. N. Dashkovskiy, and C. K. Pang, "Uniform stabilization of nonlinear systems with arbitrary switchings and dynamic uncertainties," IEEE Transactions on Automatic Control, vol. 62, no. 5, pp. 2207-2222, 2017.

[16] L. Praly and Z. P. Jiang, "Linear output feedback with dynamic high gain for nonlinear systems," Systems \& Control Letters, vol. 53, no. 2, pp. 107-116, 2004.

[17] A. Astolfi and L. Praly, "Global complete observability and output-to-state stability imply the existence of a globally convergent observer," Mathematics of Control, Signals, and Systems, vol. 18, no. 1, pp. 32-65, 2006.

[18] L. Praly, "Asymptotic stabilization via output feedback for lower triangular systems with output dependent incremental rate," IEEE Transactions on Automatic Control, vol. 48, no. 6 , pp. 1103-1108, 2003.

[19] G. Kaliora, A. Astolfi, and L. Praly, "Norm estimators and global output feedback stabilization of nonlinear systems with ISS inverse dynamics," IEEE Transactions on Automatic Control, vol. 51, no. 3, pp. 493-498, 2006.

[20] P. Krishnamurthy and F. Khorrami, "Global adaptive output feedback tracking for nonlinear systems linear in unmeasured states," in Proceeding of 2001 American Control Conference, pp. 4814-4819, Arlington, VA, USA, June 2001.

[21] M. M. Polyearpou and P. A. loannou, "A robust adaptive nonlinear control design," in Proceedings of the 1993 American Control Conference, pp. 1365-1369, San Francisco, CA, USA, June 1993.

[22] P. Krishnamurthy, F. Khorrami, and R. S. Chandra, "Global high-gain-based observer and backstepping controller for generalized output-feedback canonical form," IEEE Transactions on Automatic Control, vol. 48, no. 12, pp. 2277-2284, 2003.

[23] P. Krishnamurthy, F. Khorrami, and Z. P. Jiang, "Global output feedback tracking for nonlinear systems in generalized output-feedback canonical form," in Proceeding of the 2001 American Control Conference, pp. 4241-4246, Arlington, VA, USA, June 2001.

[24] P. Krishnamurthy and F. Khorrami, “On uniform solvability of parameter-dependent Lyapunov inequalities and applications to various problems," SIAM Journal on Control and Optimization, vol. 45, no. 4, pp. 1147-1164, 2006.

[25] F. Mazenc, L. Praly, and W. P. Dayawansa, "Global stabilization by output feedback: examples and counterexamples," Systems \& Control Letters, vol. 23, no. 2, pp. 119-125, 1994.

[26] H. Deng and M. Krstic, "Output-feedback stochastic nonlinear stabilization," IEEE Transactions on Automatic Control, vol. 44, no. 2, pp. 328-333, 1999.

[27] H. Deng and M. Krstić, "Output-feedback stabilization of stochastic nonlinear systems driven by noise of unknown covariance," Systems \& Control Letters, vol. 39, no. 3, pp. 173-182, 2000.

[28] R. Marino and P. Tomei, "Robust stabilization of feedback linearizable time-varying uncertain nonlinear systems," Automatica, vol. 29, no. 1, pp. 181-189, 1993.

[29] R. Marino and P. Tomei, "Global adaptive output-feedback control of nonlinear systems. I. linear parameterization," IEEE Transactions on Automatic Control, vol. 38, no. 1, pp. 17-32, 1993.

[30] S. Jain and F. Khorrami, "Application of a decentralized adaptive output feedback based on backstepping to power systems," in Proceedings of the 34th IEEE Conference on Decision and Control, pp. 1585-1590, New Orleans, LA, USA, December 1995.

[31] R. A. Freeman and P. V. Kokotovic, Robust Nonlinear Control Design, Birkhauser, Boston, MA, USA, 1995.

[32] Z.-P. Jiang and L. Praly, "Design of robust adaptive controllers for nonlinear systems with dynamic uncertainties," Automatica, vol. 34, no. 7, pp. 825-840, 1998.

[33] Z. G. Pan and T. Basar, "Adaptive controller design for tracking and disturbance attenuation in parametric strictfeedback nonlinear systems," IEEE Transactions on Automatic Control, vol. 43, no. 8, pp. 1066-1083, 1998. 
[34] W. Li, Y. Jing, and S. Zhang, "Output-feedback stabilization for stochastic nonlinear systems whose linearizations are not stabilizable," Automatica, vol. 46, no. 4, pp. 752-760, 2010.

[35] W. Li, X.-J. Xie, and S. Zhang, “Output-feedback stabilization of stochastic high-order nonlinear systems under weaker conditions," SIAM Journal on Control and Optimization, vol. 49, no. 3, pp. 1262-1282, 2011.

[36] L. Liu and X.-J. Xie, "Output-feedback stabilization for stochastic high-order nonlinear systems with time-varying delay," Automatica, vol. 47, no. 12, pp. 2772-2779, 2011.

[37] X.-J. Xie and W.-Q. Li, "Output-feedback control of a class of high-order stochastic nonlinear systems," International Journal of Control, vol. 82, no. 9, pp. 1692-1705, 2009.

[38] X.-J. Xie and L. Liu, "Further results on output feedback stabilization for stochastic high-order nonlinear systems with time-varying delay," Automatica, vol. 48, no. 10, pp. 2577-2586, 2012.

[39] L. C. Guo and X. K. Fang, "Bounded analysis and practical tracking control of complex stochastic nonlinear systems with unknown control coefficients," Complexity, vol. 2020, Article ID 1502065, 8 pages, 2020.

[40] F. Shang, Y. Liu, and C. Zhang, "Adaptive output feedback stabilization for a class of nonlinear systems with inherent nonlinearities and uncertainties," International Journal of Robust and Nonlinear Control, vol. 21, no. 2, pp. 157-176, 2011.

[41] F. Shang, Y. Liu, and C. Zhang, "Adaptive output feedback control for a class of planar nonlinear systems," Asian Journal of Control, vol. 11, no. 5, pp. 578-586, 2009.

[42] X. Yan and Y. Liu, "Global practical tracking for high-order uncertain nonlinear systems with unknown control directions," SIAM Journal on Control and Optimization, vol. 48, no. 7, pp. 4453-4473, 2010.

[43] X. Yan and Y. Liu, "Global practical tracking by outputfeedback for nonlinear systems with unknown growth rate," Science China Information Sciences, vol. 54, no. 10, pp. 2079-2090, 2011.

[44] X. Yan and Y. Liu, "The further result on global practical tracking for high-order uncertain nonlinear systems," Journal of Systems Science and Complexity, vol. 25, no. 2, pp. 227-237, 2012.

[45] Q. Gong and C. J. Qian, "Global practical tracking of a class of nonlinear systems by output feedback," Automatica, vol. 43, no. 1, pp. 184-189, 2007.

[46] D. Q. Mayne, S. V. Raković, R. Findeisen, and F. Allgöwer, "Robust output feedback model predictive control of constrained linear systems: time varying case," Automatica, vol. 45, no. 9, pp. 2082-2087, 2009.

[47] X. Ye, C. Li, and W. Shen, "Convergence of the variants of the Chebyshev-Halley iteration family under the Hölder condition of the first derivative," Journal of Computational and Applied Mathematics, vol. 203, no. 1, pp. 279-288, 2007.

[48] Y. Mizuta and T. Shimomura, "Sobolev's inequality for Riesz potentials with variable exponent satisfying a log-Hölder condition at infinity," Journal of Mathematical Analysis and Applications, vol. 311, no. 1, pp. 268-288, 2005. 